Pacific

Journal of

Mathematics

CURVATURES OF THE MELNIKOV TYPE, HAUSDORFF DIMENSION, RECTIFIABILITY, AND SINGULAR INTEGRALS ON R $\mathbf{R}^{n}$

HANY M. FARAG

Volume $196 \quad$ No. 2

December 2000 


\title{
CURVATURES OF THE MELNIKOV TYPE, HAUSDORFF DIMENSION, RECTIFIABILITY, AND SINGULAR INTEGRALS ON $\mathbf{R}^{n}$
}

\author{
HANY M. FARAG
}

One of the most fundamental steps leading to the solution of the analytic capacity problem (for 1-sets) was the discovery by Melnikov of an identity relating the sum of permutations of products of the Cauchy kernel to the three-point Menger curvature. We here undertake the study of analogues of this so-called Menger-Melnikov curvature, as a nonnegative function defined on certain copies of $\mathrm{R}^{n}$, in relation to some natural singular integral operators on subsets of $R^{n}$ of various Hausdorff dimensions. In recent work we proved that the Riesz kernels $x|x|^{-m-1}(m \in N \backslash\{1\})$ do not admit identities like that of Melnikov in any $L^{k}$ norm $(k \in \mathrm{N})$. In this paper we extend these investigations in various ways. Mainly, we replace the Euclidean norm $|\cdot|$ by equivalent metrics $\delta(\cdot, \cdot)$ and we consider all possible $k, m, n, \delta(\cdot, \cdot)$. We do this in hopes of finding better algebraic properties which may allow extending the ideas to higher dimensional sets. On the one hand, we show that for $m>1$ no such identities are admissible at least when $\delta$ is a norm that is invariant under reflections and permutations of the coordinates. On the other hand, for $m=1$, we show that for each choice of metric, one gets an identity and a curvature like those of Melnikov. This allows us to generalize those parts of the recent singular integral and rectifiability theories for the Cauchy kernel that depend on curvature to these much more general kernels, and provides a more general framework for the curvature approach.

\section{Introduction.}

Recall that a set $K \subset \mathbf{C}$ has vanishing analytic capacity $\gamma(K)$ if every bounded analytic function on $\mathbf{C} \backslash K$ can be extended to $\mathbf{C}$ (and is hence a constant), otherwise the set has positive analytic capacity (see $[\mathbf{A h}]$ ). In the first case the set is called removable for bounded analytic functions. Exactly which sets are removable in this sense is a very difficult question which took many decades to be answered to a reasonable extent. It is easy to show that the really interesting case is that of sets of Hausdorff 
dimension one. If the set is compact and has zero measure it is easy to show that it is then removable (see e.g., [Ma1]). It was conjectured by Denjoy that rectifiable 1 -sets (i.e., those having positive and finite measure) have positive analytic capacity. This was a consequence of Calderon's proof that the Cauchy integral operator is bounded in $L^{2}$ on Lipschitz graphs with small constant. It was also conjectured by Vitushkin [Vit] that compact purely unrectifiable 1-sets may then be removable. This was only solved recently in [Da1]. One crucial step that allowed a major breakthrough was the discovery by Melnikov [Me] of a very simple but extremely powerful identity which relates the Cauchy kernel to the classical Menger curvature $[\mathbf{B M}]$. More recently the same identity also allowed proofs that the existence of principal values of the Cauchy kernel implies rectifiability [To3], and that the boundedness of the Cauchy integral operator implies existence of principal values without doubling conditions on the measure [To2]. We explain this simple curvature idea briefly in the paragraphs below (see the survey paper [Ma3] for more details).

We recall that the so-called Menger-Melnikov curvature $c(x, y, z)$ is defined to be the reciprocal of the radius of the circle passing through $x, y, z$; $c(x, y, z)=0$ if and only if $x, y, z$ are colinear. Melnikov [Me] found the identity (for $z_{1}, z_{2}, z_{3} \in \mathbf{C}$ ):

$$
c\left(z_{1}, z_{2}, z_{3}\right)^{2}=\sum_{\sigma} \frac{1}{\left(z_{\sigma(1)}-z_{\sigma(3)}\right) \overline{\left(z_{\sigma(2)}-z_{\sigma(3)}\right)}},
$$

where the sum is over all six permutations $\sigma$ of $\{1,2,3\}$. The same identity can be transformed into $\left(x_{1}, x_{2}, x_{3} \in \mathbf{R}^{n}\right)$ :

$$
c\left(x_{1}, x_{2}, x_{3}\right)^{2}=\sum_{\sigma} \frac{\left(x_{\sigma(1)}-x_{\sigma(3)}\right) \cdot\left(x_{\sigma(2)}-x_{\sigma(3)}\right)}{\left|x_{\sigma(1)}-x_{\sigma(3)}\right|^{2}\left|x_{\sigma(2)}-x_{\sigma(3)}\right|^{2}} .
$$

It is also a simple exercise to show that

$$
c\left(x_{1}, x_{2}, x_{3}\right)^{2}=4\left\{\frac{\left|x_{1}-x_{3}\right|^{2}\left|x_{2}-x_{3}\right|^{2}-\left(\left(x_{1}-x_{3}\right) \cdot\left(x_{2}-x_{3}\right)\right)^{2}}{\left|x_{1}-x_{3}\right|^{2}\left|x_{2}-x_{3}\right|^{2}\left|x_{1}-x_{2}\right|^{2}}\right\},
$$

which, by the Schwartz inequality, shows explicitly the nonnegativity of this expression. Now suppose $\mu$ is a finite Borel measure, and that $\int \frac{\left(x_{1}-x_{3}\right)}{\left|x_{1}-x_{3}\right|^{2}} d \mu\left(x_{1}\right)$ is in $L^{2}(\mu)$. Then (ignoring any subtleties), we might formally argue that

$$
\begin{aligned}
& \frac{1}{6} \iiint c\left(x_{1}, x_{2}, x_{3}\right)^{2} d \mu\left(x_{1}\right) d \mu\left(x_{2}\right) d \mu\left(x_{3}\right) \\
& =\frac{1}{6} \iiint \sum_{\sigma} \frac{\left(x_{\sigma(1)}-x_{\sigma(3)}\right)}{\left|x_{\sigma(1)}-x_{\sigma(3)}\right|^{2}} \cdot \frac{\left(x_{\sigma(2)}-x_{\sigma(3)}\right)}{\left|x_{\sigma(2)}-x_{\sigma(3)}\right|^{2}}
\end{aligned}
$$




$$
\begin{gathered}
\cdot d \mu\left(x_{\sigma(1)}\right) d \mu\left(x_{\sigma(2)}\right) d \mu\left(x_{\sigma(3)}\right) \\
=\frac{1}{6} \sum_{\sigma} \iiint \frac{\left(x_{\sigma(1)}-x_{\sigma(3)}\right)}{\left|x_{\sigma(1)}-x_{\sigma(3)}\right|^{2}} \cdot \frac{\left(x_{\sigma(2)}-x_{\sigma(3)}\right)}{\left|x_{\sigma(2)}-x_{\sigma(3)}\right|^{2}} \\
=\int d \mu\left(x_{\sigma(1)}\right) d \mu\left(x_{\sigma(2)}\right) d \mu\left(x_{\sigma(3)}\right) \\
=\iiint \frac{\left(x_{1}-x_{3}\right)}{\left|x_{1}-x_{3}\right|^{2}} \cdot \frac{\left(x_{2}-x_{3}\right)}{\left|x_{2}-x_{3}\right|^{2}} d \mu\left(x_{1}\right) d \mu\left(x_{2}\right) d \mu\left(x_{3}\right)<\infty .
\end{gathered}
$$

This is not quite true of course, but see e.g., [MMV] where the difficulties are handled (at least for appropriate types of measures). In particular, if $\mu$ is the restriction of the one dimensional Hausdorff measure to an A-D regular set, it is proved using techniques developed in [Jo], [DS1], and [DS2] that the set is uniformly rectifiable. Combining this with a theorem of Michael Christ $[\mathbf{C h}]$, the authors prove that, if an A-D 1-set has positive analytic capacity, then it must be uniformly rectifiable. Also, Melnikov and Verdera [MV] gave a geometric proof that the Cauchy integral is bounded in $L^{2}$ on Lipschitz graphs, using this curvature. All these developments finally led to the solution of the analytic capacity problem for 1-sets by Guy David [Da1] (namely that compact totally unrectifiable 1-sets in the plane are removable for bounded analytic functions). One is then interested in doing the same for $m$ dimensional sets $(m \geq 2)$. For these the most natural analogues with the right scaling are the Riesz kernels $K_{m}=x|x|^{-m-1}$. These kernels, among others, were investigated in $[\mathbf{D S 1}],[\mathbf{D S 2}]$ on A-D regular sets, and the question of whether $\left.K_{m} * d \mathcal{H}^{1}\right|_{E} \in L^{2}(E)$ implies uniform rectifiability of the set, is still open for $m \geq 2$. Another very interesting open question of similar nature to the analytic capacity problem is whether totally unrectifiable sets are removable for Lipschitz harmonic functions. There, the only relevant sets are subsets of $\mathbf{R}^{m+1}$, having Hausdorff dimension $m \geq 2$ (for 1-sets, this was resolved in $[\mathbf{D M}]$, also making use of the Menger-Melnikov curvature). See also $[\mathrm{MPa}]$ where it is proved that a wide subclass of these sets are removable. In [Far], we proved that, for $m>1$, the most general expression arising from the Riesz kernels using $L^{k}$ norms must change sign, and hence does not produce a curvature function which measures the deviation of the set from being rectifiable. One of the results of this paper extends these results to much more general kernels. As a prototype we first study the kernels $x|x|_{p}^{-m-1}(p \geq 1)$, where for $x=\left(x^{(1)}, \cdots, x^{(n)}\right) \in \mathbf{R}^{n}$,

$$
|x|_{p} \equiv\left(\sum_{i=1}^{n}\left|x^{(i)}\right|^{p}\right)^{\frac{1}{p}} .
$$

We will however reserve the notation $|\cdot|$ for the Euclidean norm $|\cdot|_{2}$. For $m>1$, we still get the same (negative) result. However, for $m=1$, we find that these kernels give rise to curvatures equivalent to the Menger-Melnikov 
one (at least for $\infty>p>2$ ), and, hence, the same rectifiability results hold for these kernels also. We then show that most of these results actually hold for yet more general kernels of the form $k(x, y)=(x-y)(\boldsymbol{\delta}(x, y))^{-2}$, where $\boldsymbol{\delta}(\cdot, \cdot)$ is a metric equivalent to the Euclidean metric. To the best of the author's knowledge, there had previously been no (essentially) different examples, other than the Cauchy kernel, for which the curvature idea was applicable.

Acknowledgement. I would like to thank Stephen Semmes for inspiring this project, and I am very grateful to Joan Verdera for extremely valuable remarks, questions, and suggestions that significantly enhanced our results. Special thanks also to Xavier Tolsa for communicating his recent results which were quite relevant to this work.

\section{Generalizations to a new family of kernels.}

\subsection{Definitions.}

For the rest of the paper, unless otherwise stated, we will assume that $n \geq 2, k \geq 1$, are integers, and that $m \geq 1$ is a real number although the underlying measure-theoretic issue for $m \notin \mathbf{N}$ is not of interest to us since such $m$-sets are necessarily fractal in nature (see e.g., [Fe], [Fal], [Ma1]). In fact, singular integrals (at least the Riesz kernels) are typically unbounded on such sets $[\mathbf{V i h}]$. As in $[\mathbf{F a r}]$, we let $\overline{\mathcal{P}}$ be the class of all functions of the form

$P_{\boldsymbol{\delta}, m, k, n}\left(x_{0}, x_{1}, \cdots, x_{k}\right)=\sum_{\gamma \in H}\left(\frac{\sum_{j_{1}, \cdots j_{k}} a\left(j_{1}, \cdots, j_{k}\right) \prod_{i=1}^{k}\left(x_{\gamma(i)}-x_{\gamma(0)}\right)^{\left(j_{i}\right)}}{\prod_{i=1}^{k}\left(\boldsymbol{\delta}\left(x_{\gamma(i)}, x_{\gamma(0)}\right)\right)^{m+1}}\right)$,

where $x_{l} \in \mathbf{R}^{n}$ for $l=0,1, \cdots, k, \boldsymbol{\delta}(\cdot, \cdot)$ is a metric on $\mathbf{R}^{n}, H$ is the group of permutations of $\{0,1, \cdots, k\}, a:\{1, \cdots, n\}^{k} \rightarrow \mathbf{R}$, and $x_{l}^{(q)}$ denotes the $q$-th coordinate of $x_{l} \in \mathbf{R}^{n}$.

Definition 1. We say that $P_{\boldsymbol{\delta}, m, k, n}, \in \overline{\mathcal{P}}$ is a $(\boldsymbol{\delta}, m, k, n)$-curvature function of the Melnikov type, if $0 \leq P_{\boldsymbol{\delta}, m, k, n} \not \equiv 0$. 


\subsection{The one dimensional case $(m=1)$-positive results.}

2.2.1. Existence of curvatures of the Melnikov type. For $m=1$, we get positive results. The simplest and most natural norm on the transform is certainly the $L^{2}$ norm. We shall restrict to this setup for the rest of this section. We then wish to consider curvature functions of the form

$$
C_{\boldsymbol{\delta}}\left(x_{0}, x_{1}, x_{2}\right)=\sum_{\sigma \in G}\left(\frac{\sum_{i, j} a(i, j)\left(x_{\sigma(1)-} x_{\sigma(0)}\right)^{(i)}\left(x_{\sigma(2)}-x_{\sigma(0)}\right)^{(j)}}{\boldsymbol{\delta}\left(x_{\sigma(1)}, x_{\sigma(0)}\right)^{2} \boldsymbol{\delta}\left(x_{(2)}, x_{\sigma(0)}\right)^{2}}\right),
$$

where $G$ is the group of permutations of $\{0,1,2\}$. Integrals of such functions are clearly controlled by the $L^{2}$ norm of the kernels. We will also denote these curvature functions by $C_{p}$ when the metric involved is in fact the $l^{p}$ norm defined in (5). We will study this case first. As in [Far], and Section 3 below, we can reduce the existence of such curvatures to that of simpler ones with some nice properties for $a(i, j)$. Since nonnegativity is invariant under the permutations of the coordinates (and so is the norm $|\cdot|_{p}$ ), we can sum over such permutations, and then, if there are any curvatures, we can produce a curvature with $a(i, j)=a(j, i)$, for $i, j \in\{1, \cdots, n\}$. Similarly, we can assume, by invariance under the reflections $x^{(q)} \rightarrow-x^{(q)}$, for $x \in \mathbf{R}^{n}$, that $a(i, j)=0$ if $i \neq j$. By translation-invariance, we may also assume that $x_{0}=\mathbf{0}$. Setting $\mathbf{a}=x_{1}-x_{0}, \mathbf{b}=x_{2}-x_{0}$, we can assume (up to constant factor) that we have a curvature of the form

$$
C_{p}=C_{p}(\mathbf{a}, \mathbf{b}, \mathbf{0})=2\left\{\frac{\mathbf{a} \cdot \mathbf{b}}{|\mathbf{a}|_{p}^{2}|\mathbf{b}|_{p}^{2}}+\frac{(-\mathbf{a}) \cdot(\mathbf{b}-\mathbf{a})}{|\mathbf{a}|_{p}^{2}|\mathbf{b}-\mathbf{a}|_{p}^{2}}+\frac{(-\mathbf{b}) \cdot(\mathbf{a}-\mathbf{b})}{|\mathbf{b}|_{p}^{2}|\mathbf{b}-\mathbf{a}|_{p}^{2}}\right\}
$$

Let

$$
E_{p}(\mathbf{a}, \mathbf{b})=\frac{|\mathbf{a}|_{p}^{2}|\mathbf{b}|_{p}^{2}|\mathbf{b}-\mathbf{a}|_{p}^{2} C_{p}(\mathbf{a}, \mathbf{b}, \mathbf{0})}{2}
$$

We then have

$$
E_{p}(\mathbf{a}, \mathbf{b})=(\mathbf{a} \cdot \mathbf{b})\left[|\mathbf{b}-\mathbf{a}|_{p}^{2}-|\mathbf{b}|_{p}^{2}-|\mathbf{a}|_{p}^{2}\right]+|\mathbf{a}|^{2}|\mathbf{b}|_{p}^{2}+|\mathbf{b}|^{2}|\mathbf{a}|_{p}^{2} .
$$

Now the triangle inequality implies

$$
|| \mathbf{b}-\left.\mathbf{a}\right|_{p} ^{2}-|\mathbf{b}|_{p}^{2}-\left.|\mathbf{a}|_{p}^{2}|\leq 2| \mathbf{a}\right|_{p}|\mathbf{b}|_{p}
$$

So that, by the Schwarz inequality,

$$
\begin{aligned}
E_{p}(\mathbf{a}, \mathbf{b}) & \geq|\mathbf{a}|^{2}|\mathbf{b}|_{p}^{2}+|\mathbf{b}|^{2}|\mathbf{a}|_{p}^{2}-2|\mathbf{a}||\mathbf{b}||\mathbf{a}|_{p}|\mathbf{b}|_{p} \\
& \geq 0,
\end{aligned}
$$

with equality if and only if $\mathbf{a}=\lambda_{1} \mathbf{e}, \mathbf{b}=\lambda_{2} \mathbf{e}$, for some $\lambda_{1}, \lambda_{2} \in \mathbf{R}$, and $\mathbf{e} \in$ $\mathbf{R}^{n}$. 
Hence, as for the Menger-Melnikov curvature, this curvature function vanishes if and only if the three points $x_{1}, x_{2}, x_{3}$ lie on a straight line.

Set

$$
c_{p}\left(x_{1}, x_{2}, x_{3}\right)^{2} \equiv C_{p}\left(x_{1}, x_{2}, x_{3}\right),
$$

also reserving the notation $c\left(x_{1}, x_{2}, x_{3}\right)$ for $c_{2}\left(x_{1}, x_{2}, x_{3}\right)$.

Theorem 2. (1) For each choice of $p, n$, there exists a constant $A(p, n)>$ 1 such that, for all $x_{1}, x_{2}, x_{3} \in \mathbf{R}^{n}$, the following estimates hold

(a) For $p \geq 1$,

$$
c\left(x_{1}, x_{2}, x_{3}\right)^{2} \leq A(p, n) c_{p}\left(x_{1}, x_{2}, x_{3}\right)^{2} .
$$

(b) For $2 \leq p<\infty$,

$$
c_{p}\left(x_{1}, x_{2}, x_{3}\right)^{2} \leq A(p, n) c\left(x_{1}, x_{2}, x_{3}\right)^{2} .
$$

(2) For $n \geq 2$, and, either $p=\infty$, or $1 \leq p<2$, (b) fails for any choice of constant.

Proof. To prove (a) of part 1 , we assume $\mathbf{a}, \mathbf{b} \in \mathbf{R}^{n} \backslash\{\mathbf{0}\}$ are not colinear. Let $0<\varepsilon<1$ be defined via

$$
|\mathbf{a} \cdot \mathbf{b}|=(1-\varepsilon)|\mathbf{a}||\mathbf{b}| .
$$

By (9) and (10),

$$
c(\mathbf{a}, \mathbf{b}, \mathbf{0})^{2}=\frac{8 \varepsilon\left(1-\frac{\varepsilon}{2}\right)}{|\mathbf{b}-\mathbf{a}|^{2}} .
$$

On the other hand, by the same arguments leading to (12), we have

$$
c_{p}(\mathbf{a}, \mathbf{b}, \mathbf{0})^{2} \geq \frac{4 \varepsilon\left(|\mathbf{a}|^{2}|\mathbf{b}|_{p}^{2}+|\mathbf{b}|^{2}|\mathbf{a}|_{p}^{2}\right)}{|\mathbf{a}|_{p}^{2}|\mathbf{b}|_{p}^{2}|\mathbf{b}-\mathbf{a}|_{p}^{2}} .
$$

Now there exist positive constants $B(p, n)$ such that for all $x \in \mathbf{R}^{n}$,

$$
\frac{1}{B(p, n)}|x|_{p} \leq|x| \leq B(p, n)|x|_{p} .
$$

Combining (18) and (19), we obtain (a).

Now for each $p>2$, and $n \geq 2$, we fix $\varepsilon(p, n)>0$ (which we choose later). Suppose $\varepsilon \geq \varepsilon(p, n)$. Then

$$
c_{p}(\mathbf{a}, \mathbf{b}, \mathbf{0})^{2} \leq \frac{2\left(|\mathbf{a}|^{2}|\mathbf{b}|_{p}^{2}+|\mathbf{b}|^{2}|\mathbf{a}|_{p}^{2}\right)}{|\mathbf{a}|_{p}^{2}|\mathbf{b}|_{p}^{2}|\mathbf{b}-\mathbf{a}|_{p}^{2}}
$$

whereas

$$
c(\mathbf{a}, \mathbf{b}, \mathbf{0})^{2} \geq \frac{8 \varepsilon(p, n)\left(1-\frac{\varepsilon(p, n)}{2}\right)}{|\mathbf{b}-\mathbf{a}|^{2}},
$$


so that

$$
c_{p}(\mathbf{a}, \mathbf{b}, \mathbf{0})^{2} \leq\left(\frac{B(p, n)^{6}}{2 \varepsilon(p, n)\left(1-\frac{\varepsilon(p, n)}{2}\right)}\right) c(\mathbf{a}, \mathbf{b}, \mathbf{0})^{2} .
$$

Suppose now $\varepsilon<\varepsilon(p, n)$, and set $\mathbf{b}=\lambda \mathbf{a}+\mathbf{a}^{\perp}$, where

$$
\mathbf{a} \cdot \mathbf{a}^{\perp}=0 \text {. }
$$

By definition,

$$
(1-\varepsilon)^{2}|\mathbf{a}|^{2}\left\{\lambda^{2}|\mathbf{a}|^{2}+\left|\mathbf{a}^{\perp}\right|^{2}\right\}=\lambda^{2}|\mathbf{a}|^{4},
$$

so that

$$
2 \varepsilon\left(1-\frac{\varepsilon}{2}\right) \lambda^{2}|\mathbf{a}|^{2}=(1-\varepsilon)^{2}\left|\mathbf{a}^{\perp}\right|^{2} .
$$

In particular,

$$
\left|\mathbf{a}^{\perp}\right|^{2} \leq \frac{2 \varepsilon\left(1-\frac{\varepsilon}{2}\right)}{(1-\varepsilon)^{2}}|\mathbf{b}|^{2} .
$$

Now

$$
\begin{aligned}
E_{p}(\mathbf{a}, \mathbf{b})= & \lambda|\mathbf{a}|^{2}\left\{\left|(\lambda-1) \mathbf{a}+\mathbf{a}^{\perp}\right|_{p}^{2}-\left|\lambda \mathbf{a}+\mathbf{a}^{\perp}\right|_{p}^{2}-|\mathbf{a}|_{p}^{2}\right\} \\
& +\left|\lambda \mathbf{a}+\mathbf{a}^{\perp}\right|_{p}^{2}|\mathbf{a}|^{2}+|\mathbf{a}|_{p}^{2}\left\{\lambda^{2}|\mathbf{a}|^{2}+\left|\mathbf{a}^{\perp}\right|^{2}\right\} .
\end{aligned}
$$

In order to establish (b), we need to show that

$$
E_{p}(\mathbf{a}, \mathbf{b})=|\mathbf{a}|^{2}|\mathbf{b}|^{2} O(\varepsilon),
$$

with constant depending only on $p, n$. We divide this into two cases:

Case I.

$$
|1-\lambda||\mathbf{a}|_{p} \leq 10\left|\mathbf{a}^{\perp}\right|_{p}
$$

In this case, by the triangle inequality and the Schwarz inequality as before, we can group the terms in (27) in terms of either $\left|\mathbf{a}^{\perp}\right|,\left|\mathbf{a}^{\perp}\right|_{p},(1-\lambda)|\mathbf{a}|$, or $(1-\lambda)|\mathbf{a}|_{p}$ to get, (note that $\varepsilon \ll 1$ )

$$
\begin{aligned}
E_{p}(\mathbf{a}, \mathbf{b}) \leq & \lambda|\mathbf{a}|^{2}\left|(\lambda-1) \mathbf{a}+\mathbf{a}^{\perp}\right|_{p}^{2}-\lambda(1-\lambda)^{2}|\mathbf{a}|^{2}|\mathbf{a}|_{p}^{2} \\
& +2|\lambda||\lambda-1||\mathbf{a}|^{2}|\mathbf{a}|_{p}\left|\mathbf{a}^{\perp}\right|_{p}+(2+|\lambda|)|\mathbf{a}|_{p}^{2}\left|\mathbf{a}^{\perp}\right|^{2} \\
\leq & 4|\lambda||\mathbf{a}|^{2} B(p, n)^{2}\left\{400 \varepsilon|\mathbf{b}|^{2}+4 \varepsilon|\mathbf{b}|^{2}\right\} \\
& +400|\lambda| B(p, n)^{2} \varepsilon|\mathbf{a}|^{2}|\mathbf{b}|^{2}
\end{aligned}
$$




$$
+80|\lambda| B(p, n)^{2} \varepsilon|\mathbf{a}|^{2}|\mathbf{b}|^{2}+4(2+|\lambda|) B(p, n)^{2} \varepsilon|\mathbf{a}|^{2}|\mathbf{b}|^{2},
$$

where we used (26) and (29). This case will be complete if we can show that $|\lambda|$ stays bounded as $\varepsilon \rightarrow 0$ (uniformly in $\mathbf{a}, \mathbf{a}^{\perp}$ ). This however is an easy consequence of (25), (29). Suppose for instance $|\lambda|>2$. Then $|\lambda-1| \geq \frac{1}{2}|\lambda|$, and (25), (29), imply that

$$
\frac{\lambda^{2}}{4 B(p, n)^{2}}|\mathbf{a}|^{2} \leq \frac{200 \varepsilon\left(1-\frac{\varepsilon}{2}\right)}{(1-\varepsilon)^{2}} \lambda^{2}|\mathbf{a}|^{2},
$$

which is impossible for $\varepsilon$ small enough.

Case II.

$$
|1-\lambda||\mathbf{a}|_{p}>10\left|\mathbf{a}^{\perp}\right|_{p}
$$

When $\infty>p \geq 2,(27)$, and (32) allow us to expand $E_{p}(\mathbf{a}, \mathbf{b})$ in the coordinates of $\mathbf{a}^{\perp}$.

Let $\mathbf{a}=\left(a_{1}, \cdots, a_{n}\right), \mathbf{a}^{\perp}=\left(\varepsilon_{1}, \cdots, \varepsilon_{2}\right)$. We will use the expression (27) for $E_{p}$. We have already established that the constant term in the Taylor expansion must vanish. Let $L_{\mathbf{a}^{\perp}}\left(f\left(\mathbf{a}, \mathbf{a}^{\perp}\right)\right)$ denote the linear part of the Taylor expansion of a function $f\left(\mathbf{a}, \mathbf{a}^{\perp}\right)$, in terms of the coordinates of $\mathbf{a}^{\perp}$ (for fixed a). Clearly

$$
L_{\mathbf{a}^{\perp}}\left(E_{p}\right)=L_{\mathbf{a}^{\perp}}\left(F_{p}\right),
$$

where

$$
F_{p} \equiv \lambda|\mathbf{a}|^{2}\left|(\lambda-1) \mathbf{a}+\mathbf{a}^{\perp}\right|_{p}^{2}+|\mathbf{a}|^{2}(1-\lambda)\left|\lambda \mathbf{a}+\mathbf{a}^{\perp}\right|_{p}^{2}
$$

Now

$$
F_{p}=|\mathbf{a}|^{2}\left\{\lambda\left(\sum_{i=1}^{n}\left|(\lambda-1) a_{i}+\varepsilon_{i}\right|^{p}\right)^{\frac{2}{p}}+(1-\lambda)\left(\sum_{i}\left|\lambda a_{i}+\varepsilon_{i}\right|^{p}\right)^{\frac{2}{p}}\right\},
$$

and then it is easy to see that

$$
\begin{aligned}
L_{\mathbf{a}^{\perp}}\left(F_{p}\right) & =\frac{2|\mathbf{a}|^{2}|\mathbf{a}|_{p}^{2}}{|\mathbf{a}|_{p}^{p}}\left\{\sum_{i} \lambda(\lambda-1)\left|a_{i}\right|^{p-2} a_{i} \varepsilon_{i}+\sum_{i}(1-\lambda) \lambda\left|a_{i}\right|^{p-2} a_{i} \varepsilon_{i}\right\} \\
& =0 .
\end{aligned}
$$

Now let $Q_{\mathbf{a}^{\perp}}\left(f\left(\mathbf{a}, \mathbf{a}^{\perp}\right)\right)$ denote the quadratic error for $f$ in the coordinates of $\mathbf{a}^{\perp}$. Here we have

$$
Q_{\mathbf{a}^{\perp}}\left(E_{p}\right)=Q_{\mathbf{a}^{\perp}}\left(F_{p}\right)+|\mathbf{a}|_{p}^{2}\left|\mathbf{a}^{\perp}\right|^{2}
$$


so that we only need to estimate $\left|Q_{\mathbf{a}^{\perp}\left(F_{p}\right)}\right|$. We now need to compute the second partial derivatives with respect to the coordinates of $\mathbf{a}^{\perp}$. Computing, we get

$$
\begin{aligned}
& \frac{1}{2|\mathbf{a}|^{2}} \frac{\partial^{2} F_{p}}{\partial \varepsilon_{i} \partial \varepsilon_{j}} \\
& =(p-1)\left\{\begin{array}{c}
\lambda\left|(\lambda-1) a_{i}+\varepsilon_{i}\right|^{p-2}\left(\sum_{i}\left|(\lambda-1) a_{i}+\varepsilon_{i}\right|^{p}\right)^{\frac{2}{p}-1} \\
+(1-\lambda)\left|\lambda a_{i}+\varepsilon_{i}\right|^{p-2}\left(\sum_{i}\left|\lambda a_{i}+\varepsilon_{i}\right|^{p}\right)^{\frac{2}{p}-1}
\end{array}\right\} \delta_{i j} \\
& +(2-p)\left\{\begin{array}{c}
\lambda\left|(\lambda-1) a_{i}+\varepsilon_{i}\right|^{p-2}\left|(\lambda-1) a_{j}+\varepsilon_{j}\right|^{p-2} \times \\
\left.(\lambda-1) a_{j}+\varepsilon_{j}\right)\left((\lambda-1) a_{i}+\varepsilon_{i}\right)\left(\sum_{i}\left|(\lambda-1) a_{i}+\varepsilon_{i}\right|^{p}\right)^{\frac{2}{p}-2} \\
+(1-\lambda)\left|\lambda a_{i}+\varepsilon_{i}\right|^{p-2}\left|\lambda a_{j}+\varepsilon_{j}\right|^{p-2}\left(\lambda a_{i}+\varepsilon_{i}\right) \times \\
\left(\lambda a_{j}+\varepsilon_{j}\right)\left(\sum_{i}\left|\lambda a_{i}+\varepsilon_{i}\right|^{p}\right)^{\frac{2}{p}-2}
\end{array}\right\} .
\end{aligned}
$$

Hence, by (32), (36), we get the (crude) estimate

$$
E_{p} \leq|\lambda||\mathbf{a}|^{2} O\left(\left|\mathbf{a}^{\perp}\right|^{2}\right)
$$

with constant depending only on $n, p$. This is certainly sufficient if $|\lambda|$ is bounded. To estimate the case $|\lambda| \gg 1$, we estimate each of the quantities

$$
J_{i} \equiv\left\{\begin{array}{c}
\lambda\left|(\lambda-1) a_{i}+\varepsilon_{i}\right|^{p-2}\left(\sum_{i}\left|(\lambda-1) a_{i}+\varepsilon_{i}\right|^{p}\right)^{\frac{2}{p}-1} \\
+(1-\lambda)\left|\lambda a_{i}+\varepsilon_{i}\right|^{p-2}\left(\sum_{i}\left|\lambda a_{i}+\varepsilon_{i}\right|^{p}\right)^{\frac{2}{p}-1}
\end{array}\right\}
$$

and

$$
K_{i j} \equiv\left\{\begin{array}{c}
\lambda\left|(\lambda-1) a_{i}+\varepsilon_{i}\right|^{p-2}\left|(\lambda-1) a_{j}+\varepsilon_{j}\right|^{p-2} \times \\
\left((\lambda-1) a_{j}+\varepsilon_{j}\right)\left((\lambda-1) a_{i}+\varepsilon_{i}\right)\left(\sum_{i}\left|(\lambda-1) a_{i}+\varepsilon_{i}\right|^{p}\right)^{\frac{2}{p}-2} \\
+(1-\lambda)\left|\lambda a_{i}+\varepsilon_{i}\right|^{p-2}\left|\lambda a_{j}+\varepsilon_{j}\right|^{p-2}\left(\lambda a_{i}+\varepsilon_{i}\right) \times \\
\left(\lambda a_{j}+\varepsilon_{j}\right)\left(\sum_{i}\left|\lambda a_{i}+\varepsilon_{i}\right|^{p}\right)^{\frac{2}{p}-2}
\end{array}\right\} .
$$

To estimate $J_{i}, K_{i j}$, we write, for $\alpha>0$,

$$
\left|\lambda a_{i}+\varepsilon_{i}\right|^{\alpha}=|\lambda|^{\alpha}\left|a_{i}+\frac{\varepsilon_{i}}{\lambda}\right|^{\alpha}
$$


But for $|\lambda| \gg 1$, we have

$$
|\lambda|^{\alpha}\left|a_{i}+\frac{\varepsilon_{i}}{\lambda}\right|^{\alpha}=|\lambda|^{\alpha}\left|a_{i}\right|^{\alpha}\left(1+O\left(\frac{\left|\varepsilon_{i}\right|}{|\lambda|\left|a_{i}\right|}\right)+O\left(\frac{\left|\varepsilon_{i}\right|^{\alpha}}{|\lambda|^{\alpha}\left|a_{i}\right|^{\alpha}}\right)\right),
$$

and similarly,

$$
\left|(\lambda-1) a_{i}+\varepsilon_{i}\right|^{\alpha}=\left|\lambda a_{i}+\varepsilon_{i}\right|^{\alpha}\left(1+O\left(\frac{\left|a_{i}\right|}{\left|\lambda a_{i}+\varepsilon_{i}\right|}\right)+O\left(\frac{\left|\varepsilon_{i}\right|^{\alpha}}{\left|\lambda a_{i}+\varepsilon_{i}\right|^{\alpha}}\right)\right),
$$

with constants depending only on $\alpha$. Substituting these estimates back in (40), (41), we get cancellations of the highest power terms in $|\lambda|$. Namely,

$$
\begin{aligned}
\left|J_{i}\right| & \leq C^{\prime}, \\
\left|K_{i j}\right| & \leq C^{\prime \prime},
\end{aligned}
$$

with constants depending only on $n, p$. Hence,

$$
E_{p} \leq C(n, p)|\mathbf{a}|^{2}\left|\mathbf{a}^{\perp}\right|^{2} .
$$

Combining (26),(47), we obtain (20), and hence the proof of (b) is complete.

To prove Part 2 of Theorem 2 in the case $1<p<2$, a simple choice such as $\mathbf{a}=(1,0, \cdots, 0), \mathbf{b}=(2,2 \sqrt{\alpha}, 0, \cdots, 0)$, for small $\alpha$ would do. However, for $p=1$, the situation is a little more subtle. Let $\mathbf{a}=(1, \sqrt{\alpha}, 0, \cdots, 0$,$) ,$ $\mathbf{b}=\frac{3}{2}(1-\alpha, 2 \alpha, 0, \cdots, 0)$. Then

$$
\begin{aligned}
E_{p}= & \frac{3}{2}\left(1-\alpha+2 \alpha^{\frac{3}{2}}\right)\left\{\begin{array}{c}
\left(\left(\frac{1}{2}-\frac{3}{2} \alpha\right)^{p}+(\sqrt{\alpha}-2 \alpha)^{p}\right)^{\frac{2}{p}}-\left(1+\alpha^{\frac{p}{2}}\right)^{\frac{2}{p}} \\
-\frac{9}{4}\left((1-\alpha)^{p}+2^{p} \alpha^{p}\right)^{\frac{2}{p}}
\end{array}\right\} \\
& +\frac{9}{4}\left((1-\alpha)^{p}+2^{p} \alpha^{p}\right)^{\frac{2}{p}}(1+\alpha)+\frac{9}{4}\left(1+\alpha^{\frac{p}{2}}\right)^{\frac{2}{p}}\left(1-2 \alpha+5 \alpha^{2}\right) \\
= & \frac{3}{2}\left\{\frac{1}{4}\left(\frac{2}{p} 2^{p} \alpha^{\frac{p}{2}}\right)-\frac{2}{p} \alpha^{\frac{p}{2}}\right\}+\frac{9}{4}\left(\frac{2}{p} \alpha^{\frac{p}{2}}\right)+O(\alpha) \\
= & \frac{3}{p}\left(2^{p-2}+\frac{1}{2}\right) \alpha^{\frac{p}{2}}+O(\alpha) .
\end{aligned}
$$

On the other hand,

$$
\begin{aligned}
& |\mathbf{a}|^{2}|\mathbf{b}|^{2}|\mathbf{b}-\mathbf{a}|^{2} c(\mathbf{a}, \mathbf{b}, \mathbf{0})^{2} \\
& =4\left\{\frac{9}{4}(1+\alpha)\left(1-2 \alpha+5 \alpha^{2}\right)-\frac{9}{4}\left(1-\alpha+2 \alpha^{\frac{3}{2}}\right)^{2}\right\} \\
& =O(\alpha),
\end{aligned}
$$

so that (b) cannot hold for any choice of constant. For $p=\infty$, a simple choice such as $a=(1,1,0, \ldots, 0)$, and $b=\left(\frac{1}{2}+\sqrt{\alpha}, \frac{1}{2}-\sqrt{\alpha}, 0, \ldots, 0\right)$, 
gives $E_{\infty}=O(\sqrt{\alpha})$, whereas $E_{2}=O(\alpha)$. This completes the proof of Theorem 2 .

Now for a general metric $\delta$, we have (for $x_{0}, x_{1}, x_{2}$ distinct),

$$
\begin{aligned}
& C_{\boldsymbol{\delta}}\left(x_{0}, x_{1}, x_{2}\right)=\sum_{\sigma \in G}\left(\frac{\left(x_{\sigma(1)-} x_{\sigma(0)}\right) \cdot\left(x_{\sigma(2)}-x_{\sigma(0)}\right)}{\boldsymbol{\delta}\left(x_{\sigma(1)}, x_{\sigma(0)}\right)^{2} \boldsymbol{\delta}\left(x_{(2)}, x_{\sigma(0)}\right)^{2}}\right) \\
& =2\left\{\frac{\left(x_{1}-x_{0}\right) \cdot\left(x_{2}-x_{0}\right)}{\boldsymbol{\delta}\left(x_{1}, x_{0}\right)^{2} \boldsymbol{\delta}\left(x_{2}, x_{0}\right)^{2}}+\frac{\left(x_{2}-x_{1}\right) \cdot\left(x_{0}-x_{1}\right)}{\boldsymbol{\delta}\left(x_{1}, x_{2}\right)^{2} \boldsymbol{\delta}\left(x_{1}, x_{0}\right)^{2}}\right. \\
& \left.+\frac{\left(x_{1}-x_{2}\right) \cdot\left(x_{0}-x_{2}\right)}{\boldsymbol{\delta}\left(x_{1}, x_{2}\right)^{2} \boldsymbol{\delta}\left(x_{2}, x_{0}\right)^{2}}\right\}
\end{aligned}
$$

or

$$
\begin{aligned}
\boldsymbol{\delta}\left(x_{1}, x_{0}\right)^{2} \boldsymbol{\delta}\left(x_{2}, x_{0}\right)^{2} \boldsymbol{\delta}\left(x_{1}, x_{2}\right)^{2} C_{\boldsymbol{\delta}}\left(x_{0}, x_{1}, x_{2}\right) \\
\quad=2\left\{\left|x_{1}-x_{0}\right|^{2} \boldsymbol{\delta}\left(x_{2}, x_{0}\right)^{2}+\left|x_{2}-x_{0}\right|^{2} \boldsymbol{\delta}\left(x_{1,}, x_{0}\right)^{2}\right. \\
\left.+\left(x_{1}-x_{0}\right) \cdot\left(x_{2}-x_{0}\right)\left(\boldsymbol{\delta}\left(x_{1}, x_{2}\right)^{2}-\boldsymbol{\delta}\left(x_{1}, x_{0}\right)^{2}-\boldsymbol{\delta}\left(x_{2}, x_{0}\right)^{2}\right)\right\}
\end{aligned}
$$

By the Schwarz inequality and the triangle inequality as argued for the $|\cdot|_{p}$ norms, we get that $C_{\boldsymbol{\delta}}(\cdot, \cdot, \cdot)$ is indeed a curvature function which in fact vanishes only if the three points are colinear. We define $c_{\boldsymbol{\delta}}(\cdot, \cdot, \cdot)$ via

$$
c_{\boldsymbol{\delta}}(\cdot, \cdot, \cdot)^{2}=C_{\boldsymbol{\delta}}(\cdot, \cdot, \cdot) .
$$

In exactly the same way we argued for the $|\cdot|_{p}$ norms, we also get:

Theorem 3. Let $\boldsymbol{\delta}(\cdot, \cdot)$ be a metric on $\mathbf{R}^{n}$ equivalent to the Euclidean metric. Then there exists a constant $C<\infty$ (depending on the metric), such that for all distinct $x, y, z \in \mathbf{R}^{n}$,

$$
c(x, y, z)^{2} \leq C c_{\boldsymbol{\delta}}(x, y, z)^{2} .
$$

Note that Theorem 2 provided us with examples of metrics equivalent to the Euclidean one in which the opposite inequality fails with constants. For the rest of the paper we will assume that $\delta(\cdot, \cdot)$ is a metric equivalent to the Euclidean metric. In spite of the simplicity of its proof, Theorem 3 now has several important applications (this is not surprising in light of the original Melnikov identity). We will generalize a selection of the results currently known for the Cauchy kernel using curvature. 


\subsubsection{Boundedness of operators and rectifiability via curvature.} We first recall some terminology.

A positive Radon measure $\mu$ is said to have linear growth, if there exists a constant $C<\infty$ such that

$$
\mu(B) \leq C \operatorname{diam}(B),
$$

for every ball $B$. If $\mu$ also satisfies

$$
\frac{1}{C} \operatorname{diam}(B) \leq \mu(B),
$$

whenever the center of $B$ is in $\operatorname{spt}(\mu)$, then $\mu$ is said to be 1-regular or doubling. Also a set $E \subset \mathbf{R}^{n}$ is said to be an $A D$-regular 1 -set if $\left.\mathcal{H}^{1}\right|_{E}$ satisfies $(52)$, and (53), for $\operatorname{diam}(B) \leq C \operatorname{diam}(E)$. For a measure with linear growth, and a metric $\delta(\cdot, \cdot)$ equivalent to the Euclidean metric, we let $\mathcal{C}^{\delta}$ denote the singular integral operator associated with the kernel $(x-y)(\delta(x, y))^{-2}$. For $\varepsilon>0, x \in \mathbf{R}^{n}$, we set

$$
\mathcal{C}_{\varepsilon}^{\delta}(f(x) \mu)=\int_{|x-y|>\varepsilon} f(y) \frac{(x-y)}{\delta(x, y)^{2}} d \mu(y) .
$$

We then say that $\mathcal{C}^{\delta}$ is bounded on $L^{2}(\mu)$ if there exists an $M<\infty$ such that

$$
\int\left|\mathcal{C}_{\varepsilon}^{\delta}(f(x) \mu)\right|^{2} d \mu(x) \leq M \int_{E}|f|^{2} d \mu(x),
$$

for all $f \in L^{2}(\mu)$, uniformly in $\varepsilon$. If $\mu=\left.\mathcal{H}^{1}\right|_{E}$, then we say that $\mathcal{C}^{\delta}$ is bounded on $L^{2}(E)$.

Theorem 4. Let $E \subset \mathbf{R}^{n}$ be an AD-regular 1-set. Suppose that $\mathcal{C}^{\delta}$ is bounded on $L^{2}(E)$ for some metric $\delta(\cdot, \cdot)$. Then $E$ is uniformly rectifiable.

Proof. Our hypothesis implies that for any ball $B$,

$$
\int_{E \cap B}\left|\int_{E \cap B \backslash B(x, \varepsilon)} \mathbf{1}_{B} \frac{(x-y)}{\delta(x, y)^{2}} d \mathcal{H}^{1} y\right|^{2} d \mathcal{H}^{1} x \leq M^{\prime} \operatorname{diam}(B) .
$$

Proceeding as in Lemma 2.1 of [MMV], which proves this theorem in the case of the Cauchy kernel, we get

$$
\begin{aligned}
& \int_{E \cap B}\left|\int_{E \cap B \backslash B(x, \varepsilon)} \frac{(x-y)}{\delta(x, y)^{2}} d \mathcal{H}^{1} y\right|^{2} d \mathcal{H}^{1} x \\
& =\frac{1}{6} \iiint_{S_{\varepsilon}} c_{\delta}\left(x_{1}, x_{2}, x_{3}\right)^{2} d \mathcal{H}^{1} x_{1} d \mathcal{H}^{1} x_{2} d \mathcal{H}^{1} x_{3}
\end{aligned}
$$




$$
+\iiint_{T_{\varepsilon}} \frac{\left(x_{1}-x_{3}\right) \cdot\left(x_{2}-x_{3}\right)}{\delta\left(x_{1}, x_{3}\right)^{2} \delta\left(x_{2}, x_{3}\right)^{2}} d \mathcal{H}^{1} x_{1} d \mathcal{H}^{1} x_{2} d \mathcal{H}^{1} x_{3},
$$

where

$$
S_{\varepsilon}=\left\{\left(x_{1}, x_{2}, x_{3}\right) \in(E \cap B)^{3}:\left|x_{i}-x_{j}\right|>\varepsilon,\right\}
$$

and

$T_{\varepsilon}=\left\{\left(x_{1}, x_{2}, x_{3}\right) \in(E \cap B)^{3}:\left|x_{1}-x_{3}\right|>\varepsilon,\left|x_{2}-x_{3}\right|>\varepsilon,\left|x_{1}-x_{2}\right| \leq \varepsilon\right\}$.

As $\varepsilon \rightarrow 0$, the first summand tends to

$$
\frac{1}{6} \iiint_{(E \cap B)^{3}} c_{\delta}\left(x_{1}, x_{2}, x_{3}\right)^{2} d \mathcal{H}^{1} x_{1} d \mathcal{H}^{1} x_{2} d \mathcal{H}^{1} x_{3} \text {. }
$$

The integral on $S_{\varepsilon}$ is also estimated as in the $|\cdot|$ case. Namely, one has:

Lemma 5. For a positive Radon measure $\mu$ with linear growth, there exists a constant $C<\infty$, such that, for $\varepsilon>0$, and $B$ a ball,

$$
\begin{aligned}
& \mid \iiint_{\substack{x_{1}-x_{2}|>\varepsilon,\\
| x_{1}-x_{0}|>\varepsilon,\\
| x_{2}-x_{0} \mid>\varepsilon}} \frac{1}{6} c_{\delta}\left(x_{1}, x_{2}, x_{3}\right)^{2} d \mu\left(x_{1}\right) d \mu\left(x_{2}\right) d \mu\left(x_{3}\right) \\
&-\int_{B}\left|\mathcal{C}_{\varepsilon}^{\delta}\left(\chi_{B} \mu\right)\right|^{2} d \mu(x) \mid \leq C \mu(B)
\end{aligned}
$$

uniformly in $\varepsilon$.

For a proof, see the proof of (4) in [MV]. The proof there also works for our kernels and for such measures.

By Theorem 3, we conclude that there exists a constant $M<\infty$, such that for any ball $B$

$$
\iiint_{(E \cap B)^{3}} c\left(x_{1}, x_{2}, x_{3}\right)^{2} d \mathcal{H}^{1} x_{1} d \mathcal{H}^{1} x_{2} d \mathcal{H}^{1} x_{3} \leq M \operatorname{diam}(B) .
$$

By Theorem 3.6 of [MMV], which also applies to subsets of $\mathbf{R}^{n}$, we conclude that $E$ is uniformly rectifiable.

Now we have a $T(1)$ theorem for our kernels in terms of curvatures.

Theorem 6. For a positive Radon measure with linear growth, and a metric $\delta(\cdot, \cdot)$, the following are equivalent

(1) $\mathcal{C}^{\delta}$ is bounded on $L^{2}(\mu)$. 
(2) There exists a constant $C<\infty$, such that $\int_{B}\left|\mathcal{C}_{\varepsilon}^{\delta}\left(\chi_{B} \mu\right)\right|^{2} d \mu(x) \leq$ $C \mu(B)$, for every ball $B$, uniformly in $\varepsilon$.

(3) There exists a constant $C^{\prime}<\infty$, such that

$$
\iiint_{B^{3}} c_{\delta}\left(x_{1}, x_{2}, x_{3}\right)^{2} d \mu\left(x_{1}\right) d \mu\left(x_{2}\right) d \mu\left(x_{3}\right) \leq C^{\prime} \mu(B) .
$$

Proof. The equivalence of (1) and (2) for cubes is in Theorem 1.2 of [NTV2]. Alternatively, one can modify the proof in [Ve1] just by replacing $c(\cdot, \cdot, \cdot)$ by $c_{\delta}(\cdot, \cdot, \cdot$,$) which also gives the equivalence with (3)$. Obviously the equivalence of (2) and (3) follows easily from Lemma 5.

The proof of the above theorem for the Cauchy kernel was also done independently in [To1], but the arguments there are mainly complex analytic and do not generalize to these kernels. Let us denote by $\mathcal{C}^{p}\left(\right.$ instead of $\left.\mathcal{C}^{|\cdot|_{p}}\right)$ the singular integral operator associated with the metric $|\cdot|_{p}$. We can now use Theorem 6 to prove a theorem of a different nature.

Theorem 7. Suppose $\mu$ is a positive Radon measure with linear growth. If $\mathcal{C}^{\delta}$ is bounded on $L^{2}(\mu)$ for some metric $\delta$, then $\mathcal{C}^{q}$ is bounded on $L^{2}(\mu)$ for all $2 \leq q<\infty$.

Proof. This follows immediately from Theorem 2, Theorem 3 and Theorem 6.

Note that in particular this theorem shows directly that for this range of $q$, the operators $\mathcal{C}^{q}$ are bounded on uniformly rectifiable 1-sets, since the Cauchy kernel is. While this (to the best of the author's knowledge) was known (see [Da2]) mainly for $q$ an even integer or at least large $q$ due to the smoothness of the kernels which allows the usage of the method of rotations, our theorem shows that here we only need continuity of the second derivatives.

\subsubsection{Existence of principal values and rectifiability via curvature.}

In this Section we generalize some of the nice results in [To3]. First we need some more definitions and terminology. Recall that the upper density of $\mu$ at $x, \Theta_{\mu}^{*}(x)$, is defined via

$$
\Theta_{\mu}^{*}(x) \equiv \lim \sup _{r \rightarrow 0} \frac{\mu(B(x, r))}{r},
$$

similarly the lower density, $\Theta_{*, \mu}(x)$, via

$$
\Theta_{*, \mu}(x) \equiv \lim \inf _{r \rightarrow 0} \frac{\mu(B(x, r))}{r} .
$$


The pointwise curvature of a measure $\mu$ at $x, c_{\delta}^{2} \mu(x)$, is defined via

$$
c_{\delta}^{2} \mu(x) \equiv \iiint c_{\delta}^{2}(x, y, z) d \mu(y) d \mu(z),
$$

and the total curvature of $\mu, c_{\delta}^{2}(\mu)$, via

$$
c_{\delta}^{2}(\mu) \equiv \iiint c_{\delta}^{2}(x, y, z) d \mu(x) d \mu(y) d \mu(z) .
$$

The principal value of the operator $\mathcal{C}^{\delta}$ acting on $\nu$ at the point $x$, p.v. $C^{\delta} \nu(x)$, is defined via

$$
\text { p.v. } \mathcal{C}^{\delta} \nu(x) \equiv \lim _{\varepsilon \rightarrow 0} \mathcal{C}_{\varepsilon}^{\delta} \nu(x),
$$

whenever the limit exists. Also the maximal function $\mathcal{C}_{*}^{\delta} \nu(x)$ is defined via

$$
\mathcal{C}_{*}^{\delta} \nu(x) \equiv \sup _{\varepsilon>0}\left|\mathcal{C}_{\varepsilon}^{\delta} \nu(x)\right|
$$

We can now prove a theorem which combines results first proved for the Cauchy kernel in [To3].

Theorem 8. Let $\mu$ be a positive Radon measure on $\mathbf{R}^{n}$, and $\delta$ a metric. Suppose that the following condition holds:

(1) $\mathcal{C}_{*}^{\delta} \mu(x)<\infty$ and $\Theta_{\mu}^{*}(x)<\infty$, for $\mu$ a.e. $x \in \mathbf{R}^{n}$. Then

(2) there exists a countable family of Borel sets $E_{n}$ such that

$$
\operatorname{spt}(\mu)=\bigcup_{1}^{\infty} E_{n},
$$

and

$$
c_{\delta}^{2}\left(\mu \mid E_{n}\right)<\infty .
$$

Proof. We will follow the arguments in [To3] giving only the necessary changes needed for our kernels. Since $\Theta_{\mu}^{*}(x)<\infty$, for $\mu$ a.e. $x \in \mathbf{R}^{n}$, this mainly requires an analogue of Theorem 2.2 in [To3] for our operators which, in turn, is a corollary to the results of [NTV1]. The latter however work generally for our (antisymmetric) kernels provided the following standard estimate holds:

Lemma 9. If $x, y, z \in R^{n}$ are distinct and $|x-y| \leq \frac{1}{2}|y-z|$, then there is a constant $C<\infty$, depending on $\delta$, such that

$$
\left|\frac{(x-z)}{\delta(x, z)^{2}}-\frac{(y-z)}{\delta(y, z)^{2}}\right| \leq C \frac{|x-y|}{|x-z|^{2}} .
$$

The proof of Lemma 9 is straightforward using the equivalence of the metric with the Euclidean one, and the triangle inequality. Hence the same theorem also works for our operators. This gives, for any $\varepsilon>0$, a compact 
set $E_{\varepsilon} \subset \mathbf{R}^{n}$ such that $\mu\left(\mathbf{R}^{n} \backslash E_{\varepsilon}\right)<\varepsilon$, and $\mathcal{C}^{\delta}$ is bounded on $L^{2}\left(\mu \mid E_{\varepsilon}\right)$. Now by Theorem 6 , we get (2).

Theorem 8 now allows some generalizations of the corollaries which are proved for the Cauchy kernel in [To3] and which give a link to rectifiability.

Theorem 10. Let $\mu$ be a positive finite Radon measure on $\mathbf{R}^{n}$ satisfying $0<\Theta_{\mu}^{*}(x)<\infty$, for $\mu$ a.e. $x \in \mathbf{R}^{n}$. Suppose also that for some metric $\delta$, $\mathcal{C}_{*}^{\delta} \mu(x)<\infty$ for $\mu$ a.e. $x \in \mathbf{R}^{n}$. Then $\mu$ is supported on a rectifiable set.

Proof. This follows from Theorem 8, Theorem 3, and the Theorem of David and Leger $[\mathbf{L e}]$ for the Menger curvature.

Of special interest to us is the following generalization of Corollary 3.4 in [To3], which was the original motivation for generalizing the results of this section.

Theorem 11. Let $E \subset \mathbf{R}^{n}$ be $\mathcal{H}^{1}$-measurable with $0<\mathcal{H}^{1}(E)<\infty$. Suppose that for some metric $\delta$, we have that $\mathcal{C}_{*}^{\delta} d \mathcal{H}^{1}(x)<\infty$ for $\mathcal{H}^{1}$ a.e. $x \in E$, then $E$ is rectifiable. In particular, if the principal value p.v. $\mathcal{C}_{*}^{\delta} d \mathcal{H}^{1}(x)$ exists for a.e. $x \in E$, then $E$ is rectifiable.

Proof. This follows from Theorem 10, and the finiteness of the upper density for 1-sets.

Investigations of the type in Theorem 11 started (to the best of the author's knowledge) in [Ma5], later in $[\mathbf{H u}]$, and for higher dimensional sets in $[\mathrm{MPr}]$.

We mention in passing that, because we only have the one sided inequality in Theorem 3, we could not conclude some of the equivalences in [To2]. For example, a slight modification of (1), (2), and existence of principal values in Theorem 8 would be equivalent, if we knew that the operator $\mathcal{C}^{\delta}$ were bounded on $L^{2}(\mu)$ for instance (such as our operators $\mathcal{C}^{p}$ for $2 \leq p<\infty$, with $p$ even, or at least large enough $p$, for example), and that its principal values exist on Lipschitz graphs (with respect to $d \mathcal{H}^{1}$ ). In such a case one can even prove existence for principal values with respect to more general measures as in $[\mathbf{M M}]$, and [Ve2]. Also Theorem 11 would give a characterization of rectifiability in such a case. Again, this is the case for instance when $p$ is an even integer (or large $p$ ), because we already know that rectifiability implies the existence of principal values as in [Da2]. The main obstacle here is that we are allowing general metrics which are only required to be equivalent to the Euclidean one and are otherwise not smooth. There is however a limited amount of smoothness and cancellations on lines which is afforded to us by the results of $[\mathbf{K i}]$, which show that the metric is locally (almost every where) approximately a norm. It is unclear at this point whether this may suffice for our needs, and it is unclear for which metrics we may have any such a priori information. 


\section{The higher dimensional case $(m>1)$.}

The results proved for $m=1$ motivate the search for a similar approach when $m>1$. However, this was known to fail for the $L^{2}$ norm of the Cauchy kernel (see the concluding remarks in $[\mathbf{M M V}]$ ) and, more recently, for any $L^{k}$ norm of the Riesz kernels $(k \in \mathbf{N})$ in [Far]. Note that boundedness of these operators on $L^{2}(E)$ is equivalent to boundedness on $L^{r}(E)$ for all $r \in(1, \infty)$, and hence it is very useful to allow $k$ to be any positive integer. Because of the results in $[\mathbf{F a r}]$ it became necessary to allow some new degree of freedom, and allowing the $|\cdot|_{p}$ norms below was hoped to produce some positive results especially if $p$ is an even integer (suggested by Stephen Semmes). This however was not what we found. As in [Far], we will exploit the symmetries of $\mathbf{R}^{n}$ to get a handle on the class $\overline{\mathcal{P}}$. The situation here is more complicated due to the fact that, unlike the Euclidean norm $|\cdot|$, which is invariant under rotations, the norms $|\cdot|_{p}$ are not. However, the method does not completely break down but we just need to work with less symmetries. We have:

Theorem 12. Let $n \geq 2, k \geq 1$, be positive integers. If $m>1, p>0$, are real numbers, then $\left(|\cdot|_{p}, m, k, n\right)$-curvature functions of the Melnikov type do not exist.

Proof. Suppose $P_{m, k, n, p}$ is one such function. As in [Far], we also observe that $k$ must be an even integer, as can be seen by applying the transformation $x \rightarrow-x$, for all $x \in \mathbf{R}^{n}$, by which the function changes sign. Furthermore, by translation-invariance which is implicit in the form (6), we may assume that $x_{0}=\mathbf{0}$. Now we observe that, although a priori $a(\cdot, \cdots, \cdot):\{1, \cdots, n\}^{k} \rightarrow \mathbf{R}$, symmetry in the variables $x_{i}$ implies that, in fact, we can assume $a: G_{n, k} \rightarrow \mathbf{R}$, where $G_{n, k}$ is the family of finite sets $\left\{j_{1}, \cdots, j_{k}\right\}$ with length $k$, and such that each $j_{l} \in\{1, \cdots, n\}$. In other words, $a\left(j_{1}, \cdots, j_{k}\right)$ is independent of the ordering of the elements $j_{1}, \cdots, j_{k}$, but only depends on the frequencies of occurrences of the integers present besides (perhaps) the actual values of such integers also (e.g., $a(1,2,1)=a(1,1,2)=a(2,1,1))$. Furthermore, $|\cdot|_{p}$ is invariant under permutations of the coordinates, and so is nonnegativity. Thus, we can sum over such permutations, and replace $P_{m, k, n, p}$ by another function (which we give the same name), such that the resulting polynomial in the numerators in (6) will have that $a\left(j_{1}, \cdots, j_{k}\right)$ depends only on the frequencies of repetition regardless of the values of the integers that are repeated (e.g., $a(1,2,1)=a(2,3,2)=a(3,2,3)$ etc. $)$. Finally, by invariance of nonnegativity, and $|\cdot|_{p}$ under the transformations $x^{(q)} \rightarrow-x^{(q)}$, for all $x \in \mathbf{R}^{n}$, we can sum over all such transformations, and replace $P_{m, k, n, p}$ by another function (which we again denote by $P_{m, k, n, p}$ ) so that for this new function, $a\left(j_{1}, \cdots, j_{k}\right)=0$ if the frequency of occurrence of any integer is odd (e.g., 
coefficients like $a(1,2,1), a(3,3,1,2), a(3,3,3,1)$ must vanish). Note that this observation was used in the analysis in Section 2.2. Now observe that the sum over the permutations in (6) can be performed by summing functions $\mathcal{S}_{i}$, where $i=0,1, \cdots, k$, such that for $\sigma$ an element of $G$ the group of permutations of $\{1, \cdots k\}$,

$$
\mathcal{S}_{0}=\frac{\sum_{\sigma \in G} \sum_{j_{1}}^{n} \cdots \sum_{j_{k}=1}^{n} a\left(j_{1}, \cdots, j_{k}\right) \prod_{i=1}^{k} x_{\sigma(i)}^{\left(j_{i}\right)}}{\prod_{i=1}^{k}\left|x_{i}\right|_{p}^{m+1}},
$$

and $\mathcal{S}_{i}$ is obtained from $\mathcal{S}_{0}$ via the transformation $x_{i} \rightarrow-x_{i}, x_{j} \rightarrow x_{j}-x_{i}$, for $j \neq i$. Thus

$$
P_{m, k, n, p}=\sum_{i=0}^{k} \mathcal{S}_{i}
$$

It is therefore important to understand the properties of $\mathcal{S}_{0}$ first. Let us write

$$
\mathcal{S}_{0} \equiv \frac{Q_{m, k, n, p}}{\prod_{i=1}^{k}\left|x_{i}\right|_{p}^{m+1}}
$$

where

$$
Q_{m, k, n, p}=\sum_{\sigma} \sum_{j_{1}, \cdots, j_{k}} a\left(j_{1}, \cdots, j_{k}\right) \prod_{i=1}^{k} x_{\sigma(i)}^{\left(j_{i}\right)},
$$

and $a$ now satisfies all the symmetries indicated above. In particular, $a$ depends only on the frequencies of occurrences of the coordinates. Let the set of frequencies in each term be written as $\left(l_{1}, \cdots, l_{n},\right)$, where each $l_{j}$ is an even integer indicating the number of times the coordinate $j$ appears in the product. We also have

$$
\sum_{1}^{n} l_{j}=k
$$

We can then write $Q_{m, k, n, p}$ in the form

$$
\sum_{\sigma \in G} \sum_{\sum_{1}^{n} l_{j}=k} \alpha\left(l_{1}, \cdots l_{k}\right) \prod_{i=1}^{l_{1}} x_{\sigma(i)}^{(1)} \prod_{i=l_{1}+1}^{l_{1}+l_{2}} x_{\sigma(i)}^{(2)} \cdots \prod_{i=l_{1}+\cdots+l_{n-1}+1}^{l_{1}+\cdots+l_{n}} x_{\sigma(i)}^{(n)} .
$$

Let us then pick one term with a nonvanishing coefficient, and write it as

$$
\beta \prod_{i=1}^{l_{1}} x_{\sigma(i)}^{(1)} \prod_{i=l_{1}+1}^{l_{1}+l_{2}} x_{\sigma(i)}^{(2)} \cdots \prod_{i=l_{1}+\cdots+l_{n-1}+1}^{l_{1}+\cdots+l_{n}} x_{\sigma(i)}^{(n)},
$$


for some $\beta \neq 0$. By the symmetry in the coordinates discussed above, we may choose this term such that $l_{1} \neq 0$. We will now show that there are choices of points $\left\{x_{1}, \cdots, x_{k}\right\} \subset \mathbf{R}^{n}$, such that $Q_{m, k, n, p}$ assumes both negative, and positive values. We can certainly assume $\beta=1$. Let $\left\{\mathbf{e}_{i}\right\}_{i=1}^{n}$ be the canonical basis for $\mathbf{R}^{n}$. Set

$$
\begin{aligned}
x_{1} & =\mathbf{e}_{1}, \\
x_{2} & =\lambda^{2} \mathbf{e}_{2}, \\
x_{i} & =\lambda^{i} \mathbf{e}_{1},
\end{aligned}
$$

for $3 \leq i \leq l_{1}$, and, for $l_{1}+\cdots l_{r}<i \leq l_{1}+\cdots l_{r+1}$, we let

$$
x_{i}=\lambda^{i} \mathbf{e}_{r+1} \text {. }
$$

We fix this choice, and study the asymptotic behavior of $P_{m, k, n, p}$ as $\lambda \rightarrow \infty$. We first estimate the terms $\mathcal{S}_{i}$. We have

$$
\mathcal{S}_{0}=0 \text {, }
$$

since each point has a nonvanishing component in exactly one of the coordinate directions, there is an odd number of points (namely one) having a nonzero component in the $\mathbf{e}_{2}$ direction. Now we estimate $\mathcal{S}_{1}$ as an expansion in $\frac{1}{\lambda}$. Here we observe that the lowest order term comes also from the same term which we picked in (75) since for our choice of points, this give the highest power of $\lambda$ in the numerator. If $\theta=\prod_{i=1}^{n} l_{i}$ !, then, taking into account the number of ways that we can order the factors in our dominant terms,

$$
\begin{aligned}
S_{1} & =\frac{\theta\left(-\mathbf{e}_{1}\right)^{(1)}\left(\lambda^{2} \mathbf{e}_{2}-\mathbf{e}_{1}\right)^{(1)} \prod_{i=3}^{k} \lambda^{i}}{\left(\prod_{i=2}^{k} \lambda^{i}\right)^{m+1}}\left(1+O\left(\frac{1}{\lambda}\right)\right) \\
& =\frac{\theta}{\lambda^{2}\left(\prod_{i=2}^{k} \lambda^{i}\right)^{m}}\left(1+O\left(\frac{1}{\lambda}\right)\right) .
\end{aligned}
$$

Similarly,

$$
\begin{aligned}
\left|S_{2}\right| & \leq \frac{C_{2} \lambda^{2} \prod_{i=2}^{k} \lambda^{i}}{\left(\lambda^{2}\right)^{m+1}\left(\prod_{i=2}^{k} \lambda^{i}\right)^{m+1}}\left(1+O\left(\frac{1}{\lambda}\right)\right), \\
& \leq \frac{C_{2}^{\prime}}{\left(\lambda^{2 m}\right)^{2}\left(\prod_{i=3}^{k} \lambda^{i}\right)^{m}}
\end{aligned}
$$

for $\lambda \gg 1$, and, in general, for $2 \leq j \leq k$,

$$
\left|S_{j}\right| \leq \frac{C_{j}^{\prime}}{\left(\lambda^{j m}\right)^{j}\left(\prod_{i=j+1}^{k} \lambda^{i}\right)^{m}} .
$$


For $m>1$, (78)-(81) imply that $P_{m, k, n, p}$ takes positive values as $\lambda \rightarrow \infty$.

Now we set

$$
\begin{aligned}
& x_{1}=\mathbf{e}_{1}, \\
& x_{2}=-\mathbf{e}_{1}, \\
& x_{i}=\lambda^{i} \mathbf{e}_{1},
\end{aligned}
$$

for $3 \leq i \leq l_{1}$, and, for $l_{1}+\cdots l_{r}<i \leq l_{1}+\cdots l_{r+1}$, we let

$$
x_{i}=\lambda^{i} \mathbf{e}_{r+1} \text {. }
$$

In this case we have

$$
\begin{aligned}
S_{0} & =\frac{-\theta \prod_{i=3}^{k} \lambda^{i}}{\left(\prod_{i=3}^{k} \lambda^{i}\right)^{m+1}} \\
& =\frac{-\theta}{\left(\prod_{i=3}^{k} \lambda^{i}\right)^{m}}
\end{aligned}
$$

whereas, for $j=1,2$,

$$
\begin{aligned}
S_{j} & =\frac{2 \theta \prod_{i=3}^{k} \lambda^{i}}{2^{m+1}\left(\prod_{i=3}^{k} \lambda^{i}\right)^{m+1}}\left(1+O\left(\frac{1}{\lambda}\right)\right) \\
& =\frac{\theta}{2^{m}\left(\prod_{i=3}^{k} \lambda^{i}\right)^{m}}\left(1+O\left(\frac{1}{\lambda}\right)\right) .
\end{aligned}
$$

Finally, for $j \geq 3$,

$$
\left|S_{j}\right| \leq \frac{C}{\left(\lambda^{j^{2}} \prod_{i=j+1}^{k} \lambda^{i}\right)^{m}}\left(1+O\left(\frac{1}{\lambda}\right)\right),
$$

for some $C<\infty$, which is independent of $\lambda$. for $m>1$, (84)-(86) imply that $P_{m, k, n, p}$ takes negative values as $\lambda \rightarrow \infty$. This concludes the proof of Theorem 12 .

Now suppose $\|\cdot\|$ is a norm on $\mathbf{R}^{n}$ that is invariant under reflections and permutations of the coordinates (i.e., $\|x\|=\left\|x^{\prime}\right\|$, whenever $x^{\prime}$ is obtained from $x$ by permuting the coordinates $x^{(q)}$, or changing their signs). In exactly the same way we proved Theorem 12 , we can prove

Theorem 13. Let $n \geq 2, k \geq 1$, be positive integers, and $m>1$. Suppose $\|\cdot\|$ is a norm on $\mathbf{R}^{n}$ which is invariant under reflections and permutations of the coordinates. Then $(\|\cdot\|, m, k, n)$-curvature functions of the Melnikov type do not exist. 
It is worth noting that there are many other candidates for curvatures when $m>1$, which are not of the form that we considered but, still, they tend to get eliminated when subjected to an analysis similar to our proof of Theorem 13; namely sending all points far away except for three appropriately chosen points and then summing over the permutations of these points gives the dominant term which exhibits a change of sign.

\section{Concluding remarks.}

It is rather interesting that the results we found for $m$-sets seem to exhibit this kind of stability when we deform the Riesz kernels the way we did in Theorems 12 and 13, but one reasonable guess is that the behavior of higher dimensional sets may turn out to be different from that of 1-sets as far as these problems go or, at the very least, one needs another approach which avoids curvature. It is unclear whether, for $m=1$, one could associate the kernels $x|x|_{p}^{-2}$ to some function classes as is the case for bounded analytic functions, and Lipschitz harmonic functions in the plane, which are associated with the Cauchy kernel $(\zeta-z)^{-1}$ and its real version $x|x|^{-2}$ (respectively). The question of whether one kernel alone is sufficient for example in Theorem 4 was first asked in [DS1] where the boundedness of many kernels was required to conclude uniform rectifiability. The first kernel to do the job was the Cauchy kernel as first proved in [MMV]. Our results now give a large collection of candidates. Also in[DS1], the question of whether finiteness of the Cauchy maximal operator alone on a 1-set implies rectifiability was asked, and the complete answer is in [To3]. Our results generalize this to the $\mathcal{C}_{*}^{\delta}$ maximal operator. Finally, we mention in passing that it is conceivable that one may be able to use ideas relating kernels to curvatures in settings other than $\mathbf{R}^{n}$.

\section{References}

[Ah] L.V. Ahlfors, Bounded analytic functions, Duke Math. J., 14 (1947), 1-11.

[BM] L.M. Blumenthal and K. Menger, Studies in Geometry, W.H. Freeman and Company, 1970.

[Ca] A.P. Calderon, Cauchy integrals on Lipschitz curves and related operators, Proc. Nat. Acad. Sci. USA, 74 (1977), 1324-1327.

[Ch] M. Christ, A T $(b)$ theorem with remarks on analytic capacity and the Cauchy integral, Colloq. Math., 60/61 (1990), 1367-1381.

[CW] R.R. Coifman and G. Weiss, Analyse harmonique non-commutative sur certains espaces homogènes, Lecture notes in Math., 242, Springer-Verlag, 1971.

[Da1] G. David, Unrectifiable 1-sets have vanishing analytic capacity, to appear in Revista Mat. Iber.

[Da2] W W W Welets and singular integrals on curves and surfaces, Lecture Notes in Math., 1465, Springer-Verlag, Berlin, 1991. 
[DM] G. David and P. Mattila, Removable sets for Lipschitz harmonic functions in the plane, preprint, University of Jyväskylä.

[DS1] G. David and S. Semmes, Singular Integrals and Rectifiable Sets in $R^{n}$, Au-dela des graphes Lipschitziens, Asterisque, 193 (1991).

[DS2] Analysis of and on Uniformly Rectifiable Sets, Mathematical Surveys and Monographs, 38, Amer. Math. Soc., 1993.

[Fal] K.J. Falconer, Geometry of Fractal Sets, Cambridge University Press, 1985.

[Far] H.M. Farag, The Riesz kernels do not give rise to higher dimensional analogues of the Menger-Melnikov curvature, Publ. Mat., 43 (1999), 251-260.

[Fe] H. Federer, Geometric Measure Theory, Springer-Verlag, 1969.

$[\mathrm{Hu}] \mathrm{P}$. Huovinen, Existence of singular integrals and rectifiability of measures in the plane, Ann. Acad. Sci. Fenn. Ser., A I, Math. Dissertationes, 109 (1997).

[Jo] P.W. Jones, Rectifiable sets and the traveling salesman problem, Invent. Math., 102 (1990), 1-15.

[Ki] B. Kirchheim, Rectifiable metric spaces, local structure and regularity of the Hausdorff measure, Proc. AMS, 121 (1994), 113-123.

[Le] J.-C. Léger, Menger curvature and rectifiability, to appear in Ann. Math.

[Ma1] P. Mattila, Geometry of Sets and Measures in Euclidean Spaces, Cambridge University Press, 1995.

[Ma2] - On the analytic capacity and curvature of some cantor sets with non- $\sigma$ finite length, Publications Matemàtiques, 40 (1996), 195-204.

[Ma3] Singular integrals, analytic capacity and rectifiability, Journal of Fourier Analysis and Applications, Vol. 3, special issue, 1997.

[Ma4] _ Rectifiability, Analytic Capacity, and Singular Integrals, Doc. math. I. CM, II, (1998), 657-664.

[Ma5] Cauchy singular integrals and rectifiability of measures in the plane, Adv. Math., 115 (1995), 1-34.

[MM] P. Mattila and M.S. Melnikov, Existence and weak type inequalities for Cauchy integrals of general measures on rectifiable curves and sets, Proc. Amer. Math. Soc., 120 (1994), 143-149.

[MMV] P. Mattila, M.S. Melnikov and J. Verdera, The Cauchy integral, analytic capacity, and uniform rectifiability, Ann. of Math., 144 (1996), 127-136.

[MPa] P. Mattila and P.V. Paramonov, On geometric properties of harmonic Lip - $^{-}$ capacity, Pacific J. of Math., 171(2) (1995).

[MPr] P. Mattila and D. Preiss, Rectifiable measures in $R^{n}$ and existence of principal values of singular integrals, J. London Math. Soc., 52(2) (1995), 482-496.

[Me] M.S. Melnikov, Analytic capacity: Discrete approach and curvature of measure, Sbornik: Mathematics, 186(6) (1995), 827-846.

[MV] M. Melnikov and J. Verdera, A geometric proof of the $L^{2}$ boundedness of the Cauchy integral on Lipschitz graphs, Inter. Res. Not., 7 (1995), 325-331.

[NTV1] F. Nazarov, S. Treil and A. Volberg, Pulling ourselves up by the hair, preprint, 1997.

[NTV2] _ Cauchy integral and Calderon-Zygmund operators on non-homogeneous spaces, Int. Math. Res. Not., 15 (1997), 703-726. 
[NTV3] _ Weak type estimates and Cotlar inequalities for Calderon-Zygmund operators on non-homogeneous spaces, preprint, 1997.

[To1] X. Tolsa, $L^{2}$-boundedness of the Cauchy integral operator for continuous measures, to appear in Duke Math. J.

[To2] _ Cotlar's inequality and existence of the principal values for the Cauchy integral without the doubling condition, to appear in J. Reine Angew. Math.

[To3] , Principal values for the Cauchy integral and rectifiability, to appear in Proc. of Amer. Math. Soc.

[Ve1] J. Verdera, On the T(1)-Theorem for the Cauchy Integral, preprint.

[Ve2] _ A weak type inequality for Cauchy transforms of finite measures, Publ. Mat., 36 (1992), 1029-1034.

[Vih] M. Vihtilä, The boundedness of the Riesz s-transforms of measures in $R^{n}$, Proc. Amer. Math. Soc., 124 (1996), 3797-3804.

[Vit] A.G. Vitushkin, Analytic capacity of sets in problems of approximation theory, Russian Math. Surveys, 22 (1967), 139-200.

Received February 10, 1999 and revised July 1, 1999.

Department of Mathematics

California Institut of Technology

Pasadena, CA 91125

E-mail address: farag@its.caltech.edu 